\title{
Investigation and management of psittacosis in a public aviary: A One Health approach
}

\author{
Hopkins $\mathrm{J}^{1,2^{*}}$, Alsop J ${ }^{3}$, Varga $\mathrm{C}^{3}$, Pasma $\mathrm{T}^{3}$, Jekel $\mathrm{P}^{4}$, Rishi L4, Hirji MM, Filejski $\mathrm{C}^{5}$
}

\section{Abstract}

Background: Chlamydophila psittaci is a zoonotic bacterium that causes avian chlamydiosis in birds and psittacosis in people. Public aviaries provide a setting for occupational and recreational exposure to ill birds.

Objective: To describe the investigation and management of avian chlamydiosis in a public aviary in Canada using a One Health approach.

Methods: In June 2013, a previously healthy lorikeet died. Post-mortem examination showed fungal air sacculitis and hepatitis with RT-PCR testing positive for C. psittaci. Provincial veterinarians along with provincial and local public health officials investigated the avian and worker health at the aviary.

Outcome: A One Health approach to prevention, detection and response was applied to the people and birds potentially exposed to C. psittaci. The aviary was closed pending investigation and response measures. No further cases of avian or human disease were found. Birds were treated with antibiotics in the event the dead bird was infectious prior to death. The aviary was appropriately cleaned and disinfected and then re-opened to the public.

Conclusions: The C. psittaci death likely represented latent infection and/or a low virulence strain given the lack of spread to other birds or people. A coordinated, multiagency approach using a One Health framework was successfully used during the first $C$. psittaci investigation at a public aviary in Canada.

\author{
Affiliations \\ ${ }^{1}$ Hamilton Public Health Services, \\ Hamilton, ON \\ ${ }^{2}$ Clinical Epidemiology \& \\ Biostatistics, McMaster University, \\ Hamilton, ON \\ ${ }^{3}$ Office of the Chief Veterinarian \\ for Ontario, Ontario Ministry \\ of Agriculture, Food and Rural \\ Affairs, Guelph, ON \\ ${ }^{4}$ Niagara Region Public Health, \\ Thorold, ON \\ ${ }^{5}$ Ministry of Health and \\ Long-Term Care, Toronto, ON
}

${ }^{\star}$ Correspondence: jessica. hopkins@hamilton.ca

Suggested citation: Hopkins J, Alsop J, Varga C, Pasma T, Jekel P, Rishi L, et al. Investigation and management of psittacosis in a public aviary: A One Health approach. Can Comm Dis Rep 2016;42:112-6.

https://doi.org/10.14745/ccdr.v42i05a02

\section{Introduction}

On June 20, 2013, a previously healthy rainbow lorikeet (Trichoglossus haematodus) died in a public aviary in Ontario, Canada. Indoor aviaries are large enclosures that allow birds to fly and interact within a controlled environment. Public aviaries allow the public to enter the birds' habitat to view or interact with them. As per protocol, all bird deaths in the facility undergo post-mortem examination and testing. The bird's carcass was submitted to the Animal Health Laboratory, University of Guelph by the aviary's attending veterinarian. The samples sent for real-time polymerase chain reaction (RT-PCR) testing were found to be positive for Chlamydophila psittaci, suggesting active or latent infection.

\section{Background}

\section{Avian chlamydiosis and psittacosis}

Avian chlamydiosis in birds and psittacosis (ornithosis) in people are bacterial infections caused by C. psittaci (1-3). Although psittacine (parrot-type) birds, especially cockatiels and budgerigars, are most commonly infected, C. psittaci bacteria have been identified in over 460 bird species $(2,4)$. The people most at risk are bird owners or those who work with birds, although infection can occur with passing or seemingly minimal exposure (1-3). Birds with latent or clinical disease are infectious (the former intermittently), and those with clinical disease exhibit non-specific signs of lethargy, anorexia, ruffled feathers, sleepiness, shivering, weight loss, respiratory distress, serous or mucopurulent ocular or nasal discharge, conjunctivitis, diarrhea and excretion of green to yellow-green urates $(2,5)$. People with psittacosis generally present with mild flu-like symptoms, but they can develop more severe disease, including pneumonia, encephalitis and myocarditis $(1,3)$. The incubation period may be long: 3 days to several weeks in birds and 1 to 4 weeks in people $(2,6)$. Shedding of high virulence strains in birds, in particular, can lead to outbreaks $(2,3)$. The combination of asymptomatic shedding and a long incubation period makes outbreak prevention challenging (2).

In Canada, because avian chlamydiosis surveillance is not conducted nationally, the incidence is not known. In Ontario, one to two cases were diagnosed each year over the previous five at the Animal Health Laboratory, Guelph, Ontario 
(Varga C. Unpublished data; 2014). Human psittacosis is a mandatory reportable disease in Ontario (7). Between 2003 and 2007, fewer than five cases were reported yearly (6).

\section{One Health}

"One Health" is an approach to diseases that considers the interactions between people, animals and the environment (8) [Figure 1]. One Health promotes interdisciplinary work, recognizes the complementary skill sets of various disciplines (e.g., veterinary medicine, public health, human medicine, environmental health) [9] and can be used to support prevention, detection and response to public health risks (10).

\section{Rationale and objective}

A public aviary can potentially expose workers and the public to zoonoses such as psittacosis. Even a single case requires prevention of an outbreak. A framework to support a comprehensive, multiagency approach is needed. In this article, we describe the first known report of an integrated avian and human chlamydiosis/psittacosis investigation in Canada and highlight the use of One Health to describe the multiagency approach to the investigation.

\section{Methods}

The investigation of the outbreak commenced on September 18, 2013, when the lorikeet tested positive for C. psittaci, and was declared over on November 6, 2013, 44 days after the bird population in the aviary tested negative for the bacteria following antibiotic treatment. The investigation completion date was determined based on more than one maximum incubation period having passed with no further cases detected in birds or people.

\section{Aviary investigation}

The aviary was a large facility open to the public. It consisted of several areas, separated by walls and doors, with separate ventilation systems. The lorikeet that died was from the main aviary, which contained approximately 150 mostly free-flying birds. The lorikeets in this main aviary had their own cage and did not come in direct contact with other birds.

As required under the Animal Health Act (11), the Animal Health Laboratory reported the positive $C$. psittaci result in the dead lorikeet to the Ontario Ministry of Agriculture, Food and Rural Affairs (OMAFRA) who in turn notified the local public health unit, and the Ontario Ministry of Health and Long-Term Care (MOHLTC). The various agencies began working with the aviary to investigate and limit the possible spread of C. psittaci

Figure 1: Summary of One Health and its roles in the Chlamydophila psittaci investigation

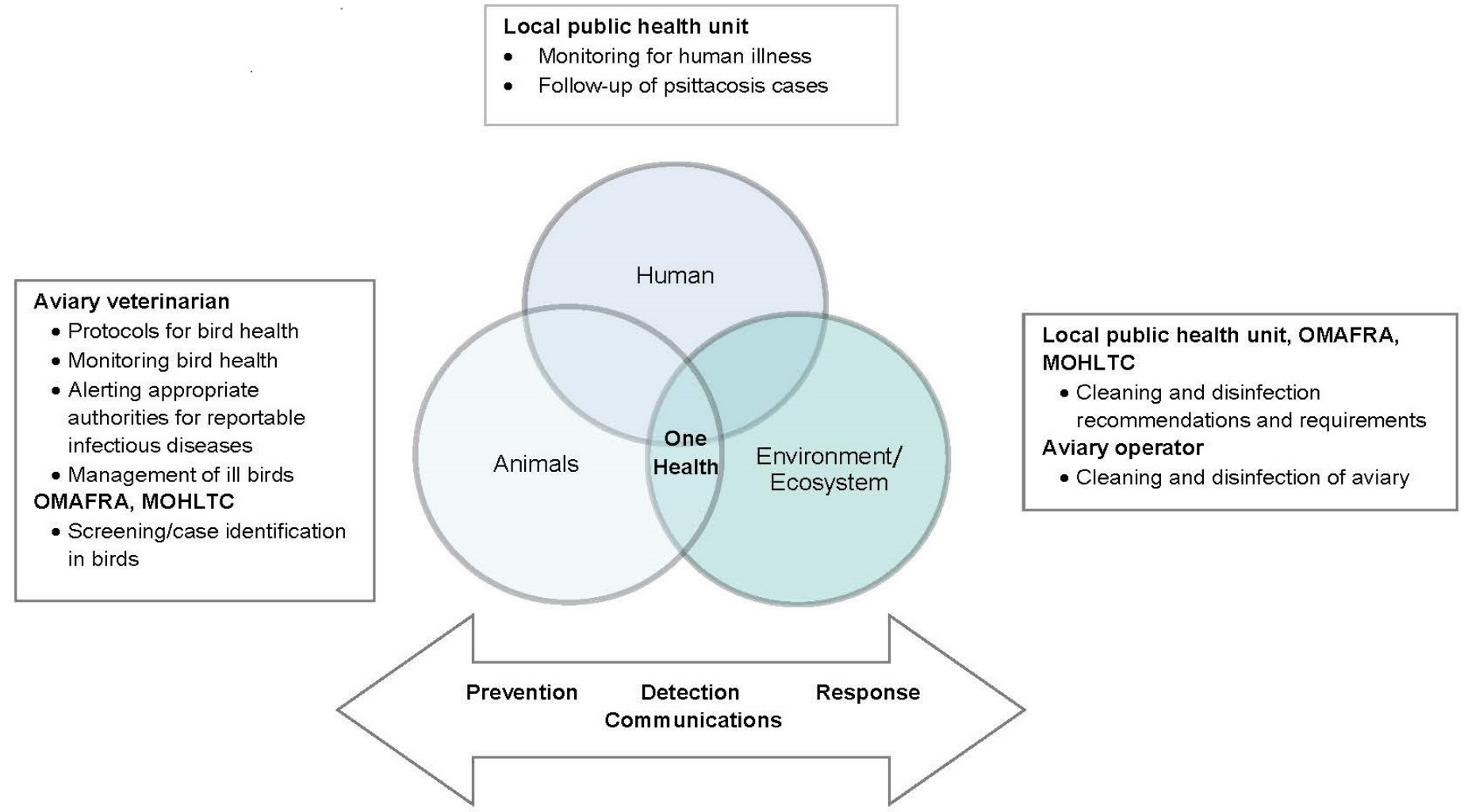

Abbreviations: MOHLTC, Ministry of Health and Long-Term Care; OMAFRA, Ontario Ministry of Agriculture, Food and Rural Affairs 
among the bird population and to people using the One Health approach of prevention, detection and response.

In order to detect $C$. psittaci in birds, veterinarians from OMAFRA and the aviary veterinarian assessed them for signs of chlamydiosis. They collected fecal samples and pharyngeal swabs from the lorikeet population and pooled fecal samples from all other species in the same air space as the lorikeets. All samples were tested for C. psittaci by RT-PCR (Animal Health Laboratory, Guelph, ON).

\section{Interventions}

Public health inspectors assessed the facility to determine the potential risk of psittacosis to visitors and to workers at the aviary and to determine the best actions to minimize or eliminate the risk of transmission from birds to people.

Three main approaches were used: 1) treating of potentially infected birds; 2) environmental cleaning of the aviary for bird secretions; and 3) restricting human contact until all birds were treated.

Birds in the aviary were treated with doxycycline for 24 days (with drug delivery in nectar for lorikeets and in water for the other birds).

Wood shavings were removed from all the cages and incinerated. A disinfectant with bactericidal, virucidal and fungicidal properties was used for the environmental cleaning of the birdcages and the parts of the exhibit open to the public. Because there are no clear guidelines, there was much discussion about disinfecting the plant life, soil, porous and irregular surfaces. Given that the lorikeets were caged and did not come in direct contact with the public, other bird species, plant life or soil, we elected to leave the plants intact, disinfect surfaces where possible and monitor for signs of human or animal illness.

Under the Animal Health Act (11), a detention order was issued stopping any movement of birds off the premises and conditions were set to incinerate all waste.

Surveillance data for respiratory syndromes in people were reviewed for signals that may have been attributable to C. psittaci. A risk assessment identified the aviary workers as having the greatest exposure and highest risk of contracting psittacosis, the public having no direct contact with birds or their cages, and so the investigation focussed on worker health. Aviary workers were educated and asked to monitor for symptoms and signs during the incubation period and report to their local public health unit if these developed.

\section{Results}

The rainbow lorikeet (Trichoglossus haematodus) that died was at least nine years old although its exact age was unknown. It was asymptomatic, and trauma was the suspected cause of death because of the observed bruising. Findings on necropsy (localized fungal air sacculitis and hepatitis) led to testing of the liver, spleen and kidney for C. psittaci and Coxiella burnetii, and the samples were positive for $C$. psittaci. No other avian deaths were reported in the facility in the month prior to notification.
The main aviary contained over 150 birds (32 lorikeets), none of which had any signs of disease. The pooled pharyngeal and fecal samples of all lorikeets were negative for C. psittaci by RT-PCR. Five pooled fecal samples taken from other bird species were also negative for $C$. psittaci. The aviary remained closed for eight days while it was cleaned and disinfected and the birds given antibiotic treatment for 48 hours. The detention order was lifted on day 44, after negative post-treatment laboratory results were received.

\section{Human investigation}

Emergency department syndromic surveillance systems did not report any activity suggestive of increased pneumonia or respiratory syndromes during the observation period.

Ten workers were identified as having had direct contact with the lorikeets or their cage and contents. No staff members were ill in the month prior to or following the death of the lorikeet and, specifically, there were no cases of significant respiratory illness. The local public health unit sent letters to all employees asking them to report any psittacosis-like illness to public health and to seek medical attention for appropriate testing and treatment. No potential cases were identified.

\section{Coordinated multiagency approach for communications}

Extensive communications took place between the provincial government ministries (OMAFRA and MOHLTC), the local public health unit, and the aviary operators and veterinarian. As this was the first known case of a possible C. psittaci outbreak in an indoor public aviary in Canada, we consulted with the Centers for Disease Control and Prevention and the National Association of Public Health Veterinarians in the United States to gain insight into their experiences with similar investigations. Given the absence of $C$. psittaci spread and lack of active or latent infection in any other birds, veterinarians and public health officials agreed that C. psittaci probably did not cause the bird's death and that appropriate steps had been taken to minimize the risk of spread in the event the bird was shedding prior to death.

\section{Discussion}

To our knowledge, this is the first report of an avian chlamydiosis outbreak investigation in a public aviary in Canada. A review of the literature found only a small number of published outbreak investigation reports worldwide. Matsui and colleagues (12) reported an outbreak of psittacosis associated with an indoor bird park in Japan, resulting in 17 human cases. Schlossberg and colleagues (13) described 13 human cases of psittacosis attributed to an aviary in a church basement. Small outbreaks have also been reported following bird shows, such as the 18 people who developed psittacosis after visiting a bird show in the Netherlands (14) and 2 confirmed (along with 46 probable and possible) cases following a bird fair in France (15). Outbreaks have also been reported in outdoor aviary settings, such as in Megellanic penguins at the San Francisco Zoo, with no human cases reported (16). These outbreaks have demonstrated that birds without clinical signs may be infectious. However, unlike the outbreaks described in previous reports, the incident we investigated did not result in an outbreak for a number of possible reasons: the bird had minimal shedding or was infected 
with a low virulence strain; the public did not have direct contact with the lorikeet; the building ventilation was adequate; and/or practices to maintain a clean aviary environment were in place.

As with any complex investigation, multiple challenges occurred and questions remained. Evidence of the survival of $C$. psittaci in the environment and the role of plant vegetation as a fomite was lacking. The aviary had significant amounts of live vegetation that regularly comes in contact with bird feces and discharges, but which visitors do not contact. In addition, the lorikeets remained in their cage and did not come in direct contact with the vegetation or the public. As mentioned previously, we elected to focus on cleaning the cages and changing the shaving materials in the lorikeet cage and left the vegetation in place suspecting its role in transmission to the public would be minimal given the lack of direct contact. We were fortunate that no further bird or human cases presented. Had this occurred, it may have been more difficult to differentiate between ongoing spread in the bird population and the role of fomites.

A full treatment course for avian chlamydiosis is usually 30 to 45 days (5). Given that this investigation focussed on a public aviary, significant business would have been lost if the aviary had remained closed for the entire duration of treatment. Economics are an important consideration in a One Health approach and, after researching the literature and consulting with an expert, we elected to allow the aviary to re-open after the birds had been treated for 48 hours, as significant organism shedding decreases after this time (5; Personal communication. Beaufrère H., Ontario Veterinary College; 2013).

Lastly, whether the dead lorikeet had active or latent infection, with implications for shedding, and the lack of experience in managing avian chlamydiosis in public aviaries led to resources being devoted to an investigation where it was likely the dead bird had latent infection that may never have led to an outbreak. Further research to understand risk factors for outbreaks of C. psittaci and most effective interventions to prevent and manage cases of $C$. psittaci would help target prevention measures and allocate resources appropriate to the response.

This investigation was strengthened by multiple jurisdictions working together using a One Health approach (Figure 1). Veterinarians detected the potential for a psittacosis outbreak and informed public health. A coordinated response to limit the spread of disease between birds and to people was initiated, which included antibiotics for birds with latent infection, infection control measures at the aviary, and surveillance for avian and human cases of infection. Finally, measures to prevent future outbreaks were considered, including ongoing surveillance in the aviary, biosecurity and ongoing quarantine procedures for birds introduced to the aviary, and improved access to alcohol-based hand rub for staff and visitors to the aviary.

By considering the animals, people and environment/ecosystem involved, One Health can provide a framework for risk minimization whereby multiple threat pathways are considered and managed, leading to a potentially more rapid or effective public health response. However, this also has a downside. Significant resources were used to investigate a single case of C. psittaci that was likely latent infection with minimal outbreak potential.
Future outbreak investigations and successful collaborations will benefit from motivated owners/operators/veterinarians working with various public officials representing human and animal health; a clear understanding of roles and responsibilities within a One Health framework; pre-existing communications protocols or channels to support collaboration; access to centralized expertise given the rarity of psittacosis outbreaks; and incorporation of further scientific evidence to support decision making as it becomes available. The use of the One Health approach, while useful in this outbreak investigation, would also benefit from analysis over time of use, and possibly the development of response protocols that may be more proportionate to assessed level of risk.

\section{Acknowledgements}

The authors thank Dr. Preeta Kutty for providing consultation during the investigation and helpful comments on the manuscript.

\section{Conflict of interest}

None.

\section{Funding}

None.

\section{References}

1. Centers for Disease Control and Prevention. Pneumonia - psittacosis. Atlanta (GA): CDC; http://www.cdc.gov/ pneumonia/atypical/psittacosis.html.

2. Canadian Centre for Occupational Health and Safety. OHS answer fact sheets - psittacosis. Ottawa (ON): CCOHS; c1997-2014. http://www.ccohs.ca/oshanswers/diseases/ psittacosis.html.

3. American Public Health Association. Psittacosis. In: Control of Communicable Diseases Manual. 19th ed. Heymann DL, editor. Washington: American Public Health Association; 2008.

4. Kaleta E, Taday EM. Avian host range of Chlamydophila spp. based on isolation, antigen detection and serology. Avian Pathol. 2003;32:435-61.

5. Smith KA, Campbell CT, Murphy J, Stobierski MG, Tengelsen LA. Compendium of measures to control Chlamydophila psittici infection among humans (psittacosis) and pet birds (avian chlamydiosis), 2010 National Association of State Public Health Veterinarians (NASPHV). J Exotic Pet Med. 2011;20:32-45.

6. Ministry of Health and Long-Term Care. Psittacosis/ ornithosis. In: Ontario Public Health Standards Infectious Diseases Protocol, 2009. Toronto (ON): MOHLTC; 2014. 
7. Health Protection and Promotion Act, R.S.O. 1990, c H.7. Government of Ontario. http://www.ontario.ca/laws/ statute/90h07.

8. Centers for Disease Control and Prevention. About One Health. Atlanta (GA): CDC. http://www.cdc.gov/onehealth/ about.html.

9. One Health Initiative. About the One Health Initiative. http://www.onehealthinitiative.com/about.php.

10. Public Health Agency of Canada. One Health. Ottawa (ON): The Agency; http://www.phac-aspc.gc.ca/owoh-umus/indexeng.php.

11. Animal Health Act, 2009, S.O. 2009, Chapter 31. Government of Ontario; http://www.ontario.ca/laws/ statute/09a31.

12. Matsui T, Nakashima K, Ohyama T, Kobayashi J, Arima $Y$, Kisimoto $T$, et al. An outbreak of psittacosis in a bird park in Japan. Epidemiol Infect. 2008;136:492-5.
13. Schlossberg D, Delgado J, Moore MM, Wishner A, Mohn J. An epidemic of avian and human psittacosis. Arch Int Med. 1993;152:2594-6.

14. Koene R, Hautvast J, Zuchner L, Voorn P, RooyackersLemmens $\mathrm{E}$, Noel $\mathrm{H}$, et al. Local cluster of psittacosis after bird show in the Netherlands, November 2007. Euro Surveill. 2007;12:E071213.1.

15. Belchior E, Barataud D, Ollivier R, Capek I, Laroucau K, de Barbeyrac $B$, et al. Psittacosis outbreak after participation in a bird fair, Western France, December 2008. Epidemiol Infect. 2008;139:1637-41.

16. Jencek JE, Beaufrère H, Tully TN Jr, Garner MM, Dunker $\mathrm{FH}$, Baszler TV. An outbreak of Chlamydophila psittaci in an outdoor colony of Magellanic penguins (Spheniscus magellanicus). J Avian Med Surg. 2012;26:225-31. 